\title{
Evaluation of Small-Animal PET Outcome Measures to Detect Disease Modification Induced by BACE Inhibition in a Transgenic Mouse Model of Alzheimer Disease
}

\author{
Steven Deleye ${ }^{1}$, Ann-Marie Waldron ${ }^{1}$, Jeroen Verhaeghe ${ }^{1}$, Astrid Bottelbergs ${ }^{2}$, Leonie Wyffels ${ }^{1,3}$, Bianca Van Broeck ${ }^{2}$, \\ Xavier Langlois ${ }^{4}$, Mark Schmidt ${ }^{2}$, Sigrid Stroobants ${ }^{3}$, and Steven Staelens ${ }^{1}$ \\ ${ }^{I}$ Molecular Imaging Center Antwerp, University of Antwerp, Antwerp, Belgium; ${ }^{2}$ Neuroscience Department, Janssen Pharmaceutica \\ NV, Beerse, Belgium; ${ }^{3}$ Nuclear Medicine Department, University Hospital Antwerp, Antwerp, Belgium; and ${ }^{4}$ Foundational \\ Neuroscience Center, Abbvie, Cambridge, Massachusetts
}

In this study, we investigated the effects of chronic administration of an inhibitor of the $\beta$-site amyloid precursor protein-cleaving enzyme 1 (BACE1) on Alzheimer-related pathology by multitracer PET imaging in transgenic APPPS1-21 (TG) mice. Methods: Wild-type (WT) and TG mice received vehicle or BACE inhibitor $(60 \mathrm{mg} / \mathrm{kg})$ starting at 7 wk of age. Outcome measures of brain metabolism, neuroinflammation, and amyloid- $\beta$ pathology were obtained through small-animal PET imaging with ${ }^{18} \mathrm{~F}-\mathrm{FDG},{ }^{18} \mathrm{~F}$-peripheral benzodiazepine receptor ( $\left.{ }^{18} \mathrm{~F}-\mathrm{PBR}\right)$, and ${ }^{18} \mathrm{~F}$-florbetapir ( $\left.{ }^{18} \mathrm{~F}-\mathrm{AV} 45\right)$, respectively. Baseline scans were acquired at $6-7 \mathrm{wk}$ of age and follow-up scans at 4, 7, and 12 mo. ${ }^{18} \mathrm{~F}-\mathrm{AV} 45$ uptake was measured at 8 and 13 mo of age. After the final scans, histologic measures of amyloid- $\beta$ (4G8), microglia (ionized calcium binding adaptor molecule 1), astrocytes (glial fibrillary acidic protein), and neuronal nuclei were performed. Results: TG mice demonstrated significant age-associated increases in ${ }^{18} \mathrm{~F}-\mathrm{AV} 45$ uptake. An effect of treatment was observed in the cortex $(P=0.0014)$, hippocampus $(P=0.0005)$, and thalamus $(P<0.0001)$. Histology confirmed reduction of amyloid- $\beta$ pathology in TG-BACE mice. Regardless of treatment, TG mice demonstrated significantly lower ${ }^{18} \mathrm{~F}-\mathrm{FDG}$ uptake than WT mice in the thalamus $(P=0.0004)$ and hippocampus $(P=0.0332)$. Neuronal nucleus staining was lower in both TG groups in the thalamus and cortex. ${ }^{18} \mathrm{~F}-\mathrm{PBR} 111$ detected a significant age-related increase in TG mice $(P<0.0001)$ but did not detect the treatment-induced reduction in activated microglia as demonstrated by histology. Conclusion: Although ${ }^{18} \mathrm{~F}-\mathrm{FDG},{ }^{18} \mathrm{~F}-\mathrm{PBR} 111$, and ${ }^{18} \mathrm{~F}-\mathrm{AV} 45$ all detected pathologic alterations between TG and WT mice, only ${ }^{18} \mathrm{~F}-\mathrm{AV} 45$ could detect an effect of BACE inhibitor treatment. However, changes in WT binding of ${ }^{18} \mathrm{~F}-\mathrm{AV} 45$ undermine the specificity of this effect.

Key Words: small animal PET; Alzheimer's disease; longitudinal; BACE inhibitor

J Nucl Med 2017; 58:1977-1983

DOI: 10.2967/jnumed.116.187625

\footnotetext{
A lzheimer disease is a progressive neurodegenerative disease pathologically characterized by amyloid- $\beta$ plaques, neurofibrillary tangles, inflammation, and neuronal loss (1). Molecular imaging

Received Dec. 6, 2016; revision accepted May 31, 2017

For correspondence or reprints contact: Steven Staelens, Molecular Imaging Center Antwerp, University of Antwerp, Campus Drie Eiken-UC, Universiteitsplein 1, 2610 Wilrijk, Belgium.

E-mail: steven.staelens@uantwerpen.be

Published online Jun. 13, 2017.

COPYRIGHT (c) 2017 by the Society of Nuclear Medicine and Molecular Imaging.
}

techniques such as PET are powerful tools to noninvasively monitor these pathologic features and are equally applicable in humans and animal models. Amyloid plaques are imaged using PET tracers, such as ${ }^{18} \mathrm{~F}$-florbetapir $\left({ }^{18} \mathrm{~F}\right.$-AV45), which bind to the $\beta$-pleated sheet structure in these deposits (2). Inflammation can be assessed using agents targeting the mitochondrial membrane $18-\mathrm{kDa}$ translocator protein (3), and the glucose analog ${ }^{18} \mathrm{~F}-\mathrm{FDG}$ is used as a surrogate marker of neural activity to monitor neuronal loss (4).

Being noninvasive, PET enables repeated measures of animals at baseline and continually during disease and treatment, thus presenting an ideal drug-screening tool with high translatability to the clinic. The objective of this study was to investigate the effects of chronic administration of a $\beta$-site amyloid precursor protein (APP)-cleaving enzyme (BACE) inhibitor (JNJ-49146981) (5) on Alzheimer disease-related pathology by multitracer smallanimal PET imaging in transgenic APPPS1-21 (TG) mice. The amyloidogenic pathway is initiated through proteolytic processing of amyloid precursor protein by BACE1. Inhibition of BACE1 activity by genetic (6-8) or pharmacologic (9-13) means strongly supports a therapeutic benefit demonstrating reductions in amyloid- $\beta$ levels and rescued cognitive deficits in animal models, and several clinical trials of BACE inhibitors are under way (14). TG mice recapitulate certain features of Alzheimer disease, including amyloid- $\beta$ deposition, gliosis, loss of dendritic spines, and cognitive deficits (15-17). Wild-type (WT) and TG mice underwent treatment with vehicle or BACE inhibitor, and outcome measures of amyloid pathology, brain function, and neuroinflammation were obtained through small-animal PET imaging with ${ }^{18} \mathrm{~F}-\mathrm{FDG}$, ${ }^{18} \mathrm{~F}$-peripheral benzodiazepine receptor $\left({ }^{18} \mathrm{~F}-\mathrm{PBR}\right) 1$, and ${ }^{18} \mathrm{~F}-\mathrm{AV} 45$, respectively. Although the effects of an antiamyloid antibody (18) and a $\gamma$-secretase modulator (19) have been previously evaluated with small-animal PET imaging, to our knowledge this is the first report of small-animal PET monitoring of BACE inhibition.

\section{MATERIALS AND METHODS}

\section{Animals}

Animals were treated in accordance with the European Ethics Committee (decree 86/609/CEE) with the approval of the local Animal Experimental Ethical Committee of the University of Antwerp, Belgium (2014-65). The animals were group-housed, 7 per cage, under environmentally controlled conditions (12-h light-dark cycle, $20^{\circ} \mathrm{C}-24^{\circ} \mathrm{C}$, and $40 \%-70 \%$ relative humidity) in individually ventilated cages with food (ssniff R/M-H; Bio Services) and water ad libitum. TG mice coexpress the human Swedish double amyloid precursor protein mutation 
KM670/671NL and the human mutated PS1 L166P driven by the neuron-specific Thy-1 promoter. $A \beta$ deposition begins as early as $6 \mathrm{wk}$ of age, but no mature tau tangles are formed (15). Also, activated microglia surrounding the deposits, as well as increased astrogliosis, are present at $6 \mathrm{wk}$ (15). Age-matched C57BL/6 littermates were used as controls. All mice $(n=112)$ were female and were received in kind from Janssen Pharmaceutica NV.

\section{Treatment}

JNJ-49146981 was synthesized at Janssen Pharmaceutica as described in patent literature (5). The chemical structure of JNJ-49146981 is shown in Supplemental Fig. 1 (supplemental materials are available at http://jnm.snmjournals.org). JNJ-49146981 is a potent brain-penetrating BACE inhibitor with a half-maximal inhibitory concentration of $4.6 \mathrm{nM}$ in an enzymatic BACE1 assay and $0.9 \mathrm{nM}$ in a cellular assay measuring reduction in A $\beta 42$ levels (20). Further pharmacokinetic details are provided in the supplemental materials.

TG mice $(n=56)$ and their WT littermates $(n=56)$ were randomly divided into 2 groups to receive either the BACE inhibitor $(60 \mathrm{mg} / \mathrm{kg})$ or vehicle treatment, resulting in 4 groups of 28 mice each: WT-VEH, WT-BACE, TG-VEH, and TG-BACE. Vehicle mice received a $40 \%$ Captisol (Cydex Pharmaceuticals) solution, prepared by dissolving $40 \mathrm{~g}$ of Captisol in $100 \mathrm{~mL}$ of sterile water. BACE inhibitor solution was prepared by dissolving $60 \mathrm{mg}$ of the active compound in $0.5 \mathrm{~mL}$ of $1 \mathrm{~N}$ acetic acid, $5 \mathrm{~mL}$ of $40 \%$ Captisol solution, and $4.5 \mathrm{~mL}$ of sterile water. Both treatments were initiated at $7 \mathrm{wk}$ of age and applied daily (for $\sim 12 \mathrm{mo}$ ) via oral gavage with a stainless steel feeding tube (20 gauge $\times 38 \mathrm{~mm}$; Instech Laboratories).

\section{Image Acquisition and Processing}

An 18- to $19-\mathrm{MBq}$ injection of radiotracer was administered through the tail vein (maximum volume, $200 \mu \mathrm{L}$ ) to awake mice. Before scanning, the animals were anesthetized by inhalation of isoflurane (5\% for induction; $2 \%$ for maintenance during preparation and scanning) supplemented with oxygen. The following imaging protocols were used, depending on the radiotracer: a 45-min uptake period followed by a 20 -min scan for ${ }^{18} \mathrm{~F}$-FDG, a 40 -min uptake period and 20-min scan for ${ }^{18} \mathrm{~F}-\mathrm{PBR} 111$, and a 30 -min uptake period and 20-min scan for ${ }^{18} \mathrm{~F}-\mathrm{AV} 45$. The animals were awake during the uptake period. For ${ }^{18}$ F-FDG, the animals were kept fasting overnight $(10-14 \mathrm{~h})$ before scanning, and plasma glucose levels were measured using a blood glucose meter (One Touch Ultra 2; LifeScan) immediately before tracer injection.

Tracer Radiosynthesis. ${ }^{18} \mathrm{~F}-\mathrm{AV} 45$ was synthesized from the tosyloxy precursor AV-105 by modification of the method described by Yao et al. (21) on a FluorSynthon I synthesis module (Comecer Netherlands) that was adapted for fully automated production of ${ }^{18} \mathrm{~F}-\mathrm{AV} 45 .{ }^{18} \mathrm{~F}-\mathrm{FDG}$ was prepared using a cassette-based FASTlab synthesis module (GE Healthcare) and was diluted with $0.9 \% \mathrm{NaCl}$ for injection. ${ }^{18} \mathrm{~F}$-PBR111 was prepared from the $p$-toluenesulfonyl precursor using automated methods on a Tracerlab $\mathrm{FX}_{\mathrm{FN}}$ (GE Healthcare) by adapting a previously described procedure (22).

PET Imaging and Analysis. All PET imaging was performed in a head-to-head configuration. The respiration rate and body temperature of the animal were constantly monitored during the entire scanning period. The core body temperature was maintained by flowing heated air through the imaging cell. PET imaging was performed using an Inveon PET/CT scanner (Siemens Preclinical Solutions) (23). The scanner uses $1.59 \times 1.59 \times 10 \mathrm{~mm}$ lutetium oxyorthosilicate crystals grouped into blocks of $20 \times 20$ crystals. The energy and timing window were set to $350-650 \mathrm{keV}$ and 3.432 ns, respectively. The PET images were reconstructed using 2-dimensional ordered subset expectation maximization (24) with 4 iterations and 16 subsets after Fourier rebinning (25). Corrections
TABLE 1

Number of Animals in Each Treatment Group Scanned at Each Time Point

\begin{tabular}{lcccc}
\hline Time point & WT-VEH & TG-VEH & WT-BACE & TG-BACE \\
\hline 6-7 wk & 28 & 28 & 28 & 28 \\
4 mo & 28 & 24 & 28 & $26^{\star}$ \\
7-8 mo & 28 & 23 & 28 & $24^{\dagger}$ \\
$12-13$ mo & 27 & 20 & 27 & 22
\end{tabular}

${ }^{*}$ One mouse underwent ${ }^{18} \mathrm{~F}-\mathrm{PBR} 111$ scan but died before ${ }^{18} \mathrm{~F}-\mathrm{FDG}$ scan.

${ }^{\dagger}$ One mouse underwent both ${ }^{18} \mathrm{~F}-\mathrm{PBR} 111$ and ${ }^{18} \mathrm{~F}-\mathrm{FDG}$ scans but died before ${ }^{18} \mathrm{~F}-\mathrm{AV} 45$ scan.

were applied for normalization, dead time, randoms, CT-based attenuation, and single-scatter simulation, as developed by Watson et al. (26). PET images were reconstructed on a $128 \times 128 \times 159$ grid with a pixel size of $0.776 \mathrm{~mm}$ and a slice thickness of $0.796 \mathrm{~mm}$. CT imaging was done using a $220^{\circ}$ rotation with 120 rotation steps. Voltage and amperage were set to $80 \mathrm{keV}$ and $500 \mu \mathrm{A}$, respectively. The CT images were reconstructed using the Feldkamp filtered-backprojection algorithm.

Image Processing. Images were processed using PMOD software (version 3.6; PMOD Technologies). Each individual PET image was transformed into the space of a predefined mouse brain template (27) by matching the individual CT images to the CT images of the template and applying the same transformation to the subject's PET images. These spatially normalized images were then analyzed using an MRI mouse whole-brain volume-of-interest template (27) available in the same software package and a priori coregistered to the mouse brain CT template. Volume-of-interest statistics in $\mathrm{kBq} / \mathrm{cm}^{3}$ were generated in the cortex, thalamus, and hippocampus and used to calculate the SUV (average tissue activity concentration $\left[\mathrm{kBq} / \mathrm{cm}^{3}\right] /$ injected dose $[\mathrm{kBq}] /$ body weight $[\mathrm{g}]$ ) for ${ }^{18} \mathrm{~F}-\mathrm{PBR} 111$ and ${ }^{18} \mathrm{~F}-\mathrm{AV} 45$ imaging data. SUVs were corrected for blood glucose levels $\left(\mathrm{SUV}_{\mathrm{glc}}, \mathrm{mg} / \mathrm{dL}\right)$ for the ${ }^{18} \mathrm{~F}-\mathrm{FDG}$ imaging data.

\section{Immunohistochemistry}

Staining was performed with 4G8, ionized calcium-binding adaptor molecule 1 (Iba-1), glial fibrillary acidic protein (GFAP), and neuronal nuclei $(\mathrm{NeuN})$.

The mice were sacrificed by decapitation, after which the brains were removed from the skull, fixed overnight in a formalin-based fixative, embedded in paraffin, and sliced $(5 \mu \mathrm{m})$ with a microtome. Sections were dewaxed with xylene and rehydrated by submerging in a graded series of ethanol with decreasing concentrations. For sections to be stained with the 4G8 antibody, antigen was retrieved in $70 \%$ formic acid (diluted in distilled water). For sections to be stained for NeuN and GFAP, heat-induced antigen retrieval was performed in citrate buffer ( $\mathrm{pH}$ 6.0). For all sections, endogenous peroxidase activity was blocked with $3 \%$ hydrogen peroxide. Samples were incubated for $1 \mathrm{~h}$ with primary antibodies (Table 1) in antibody diluent with background-reducing components (DAKO). After extensive washing, peroxidase-labeled antimouse secondary antibody (Envision; DAKO) was applied for $30 \mathrm{~min}$, followed by chromogenic labeling with 3,3-diaminobenzidine (DAKO). The slides were counterstained with hematoxylin, dehydrated, and permanently mounted (Vectamount; Vector Labs).

Imaging was performed with a NanoZoomer slide scanner (Hamamatsu Photonics) and analyzed with Matlab/Phaedra. Regions of interest 


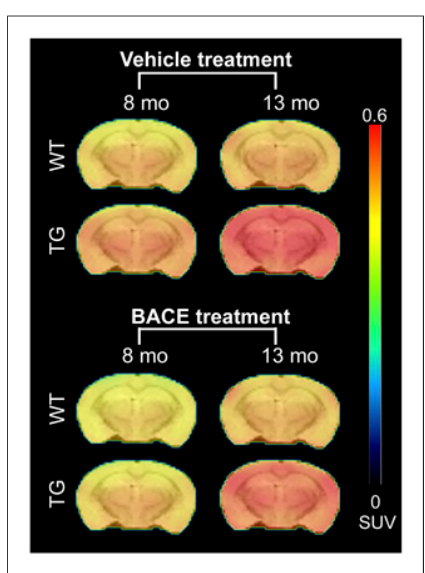

FIGURE 1. Average PET images of ${ }^{18} \mathrm{~F}$-AV45 uptake in all animal groups, overlaid on MRI T2 template for anatomic reference. were manually delineated in accordance with the Franklin and Paxinos atlas (28), and for each region of interest the percentage of 3,3-diaminobenzidine-labeled area per total area was calculated. For microglia (Iba1), two thresholds were defined, one including total Iba1 labeling (resting + activated microglia) and one taking only activated microglia into account (Supplemental Fig. 2).

\section{Statistical Analysis}

Statistical analysis was conducted in JMP Pro (version 12; SAS Institute Inc.) and GraphPad Prism (version 6; GraphPad Software). We used a linear mixed model to investigate the relationship between tracer uptake and genotype, age, and treatment. Age, genotype, and treatment were investigated as fixed effects, and mouse identification number was incorporated as a random intercept to model subject-specific responses. We additionally tested for genotype $\times$ age and between genotype $\times$ treatment interactions in this model. A quadratic age variable $\left(\mathrm{age}^{2}\right)$ was included in the analysis of ${ }^{18} \mathrm{~F}$-FDG to account for a curvilinear response. The correlation between small-animal PET data and ex vivo histology was analyzed using Pearson correlation. Histology was analyzed
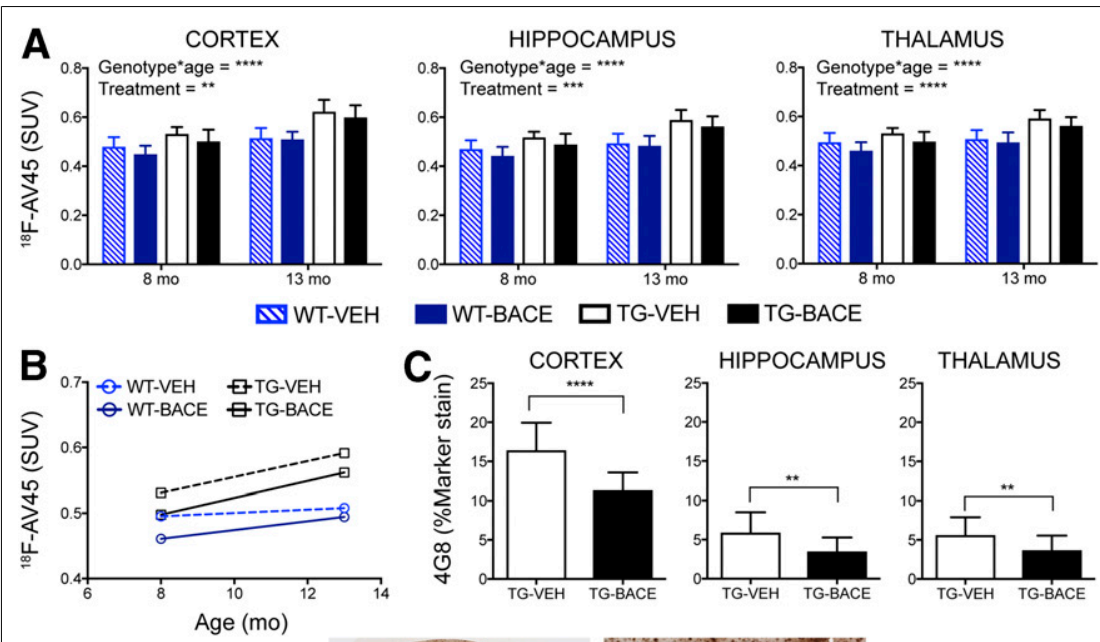

D

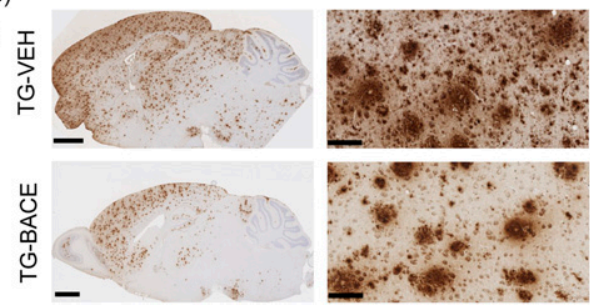

FIGURE 2. Progression of ${ }^{18} \mathrm{~F}-\mathrm{AV} 45$ uptake and its relationship to $4 \mathrm{G} 8$ staining. (A) ${ }^{18} \mathrm{~F}-\mathrm{AV} 45$ uptake (mean $+\mathrm{SD}$ ) in cortex, hippocampus, and thalamus. (B) Longitudinal trajectory of mean ${ }^{18}$ F-AV45 uptake in thalamus. (C) 4G8 staining for amyloid- $\beta$ (mean + SD) in cortex, hippocampus, and thalamus. (D) Representative images of $4 G 8$ staining in TG mice. Scale bar $=1.25 \mathrm{~mm}$ (left) and $100 \mu \mathrm{m}$ (right: cortex). ${ }^{\star} P<0.05 ;{ }^{\star \star} P<0.01$; ${ }^{\star \star \star} P<0.001$; ${ }^{\star \star \star \star} P<0.0001$.

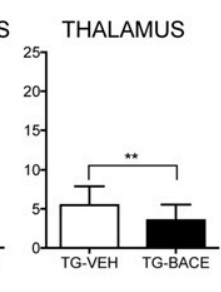

with either a 2-way ANOVA or an unpaired Student or Welch $t$ test. The Cohen d statistic was calculated to compare effect sizes between histologic and PET measures of microglia and amyloid burden in TG-VEH and TG-BACE mice. Significance levels are given to 4 decimal points.

\section{RESULTS}

Animals

During the study, 1 WT-VEH mouse was euthanized because of body weight issues. In addition, 6 TG-BACE mice, 8 TG-VEH mice, and 1 WT-BACE mouse died spontaneously. The number of animals in each treatment group for every time point is shown in Table 1. BACE-treated animals developed hair depigmentation manifesting as gray patches of fur, as has been previously described (29).

\section{${ }^{18}$ F-AV45 Imaging}

Figure 1 shows the average ${ }^{18} \mathrm{~F}$-AV45 uptake per animal group at 8 and 13 mo. Graphical analysis of ${ }^{18} \mathrm{~F}-\mathrm{AV} 45$ uptake is shown in Figures $2 \mathrm{~A}$ and $2 \mathrm{~B}$. A significant interaction of genotype $\times$ age on ${ }^{18} \mathrm{~F}-\mathrm{AV} 45$ was detected in the cortex, thalamus, and hippocampus (all $P<0.0001$ ), resulting from increasing ${ }^{18} \mathrm{~F}$-AV45 uptake from 8 to $13 \mathrm{mo}$. A significant effect of treatment was observed in the cortex $(P=0.0014)$, thalamus $(P<0.0001)$, and hippocampus $(P=0.0005)$. A genotype $\times$ treatment interaction was not observed.

4G8 staining demonstrated a clear reduction in plaque load in TG-BACE mice, as shown in Figure 2C. This reduction was significant in the cortex $(P<0.0001$, Cohen $\mathrm{d}=1.7166)$, thalamus $(P=0.0043$, Cohen $\mathrm{d}=0.9302)$, and hippocampus $(P=0.0016$, Cohen $\mathrm{d}=1.8255$ ). Minimal plaque staining was observed in the cerebellum of TG mice. There was a moderate positive correlation between $4 \mathrm{G} 8$ staining and ${ }^{18} \mathrm{~F}-\mathrm{AV} 45$ uptake ( $r=0.4686, P<0.0001)$. Representative images of 4G8 staining in TG mice are shown in Figure 2D. The effect sizes for differences in ${ }^{18} \mathrm{~F}-\mathrm{AV} 45$ uptake between TG-VEH and TG-BACE mice at 13 mo were lower than those detected with histology, with Cohen $\mathrm{d}=0.4742,0.8168$, and 0.6585 for the cortex, thalamus, and hippocampus, respectively.

\section{${ }^{18}$ F-FDG Imaging}

Figure 3 shows the average ${ }^{18} \mathrm{~F}-\mathrm{FDG}$ uptake per animal group for each time point, and graphical analysis of ${ }^{18} \mathrm{~F}-\mathrm{FDG}$ uptake is shown in Figures 4A and 4B. Age had a significant linear $(P<0.0001)$ and quadratic $(P<0.0001)$ effect on ${ }^{18} \mathrm{~F}-\mathrm{FDG}$ in all regions, supporting an age-related increase in tracer uptake that becomes less pronounced with time. Genotype had a significant main effect on ${ }^{18} \mathrm{~F}$-FDG uptake in the thalamus $(P=0.0004)$ and hippocampus $(P=0.0332)$, with TG mice demonstrating lower ${ }^{18}$ F-FDG uptake in these regions than WT mice. No genotype $\times$ age or genotype $\times$ age $^{2}$ interaction was found, showing that age-related changes in ${ }^{18}$ F-FDG uptake were not affected by genotype. No effect of treatment and no 


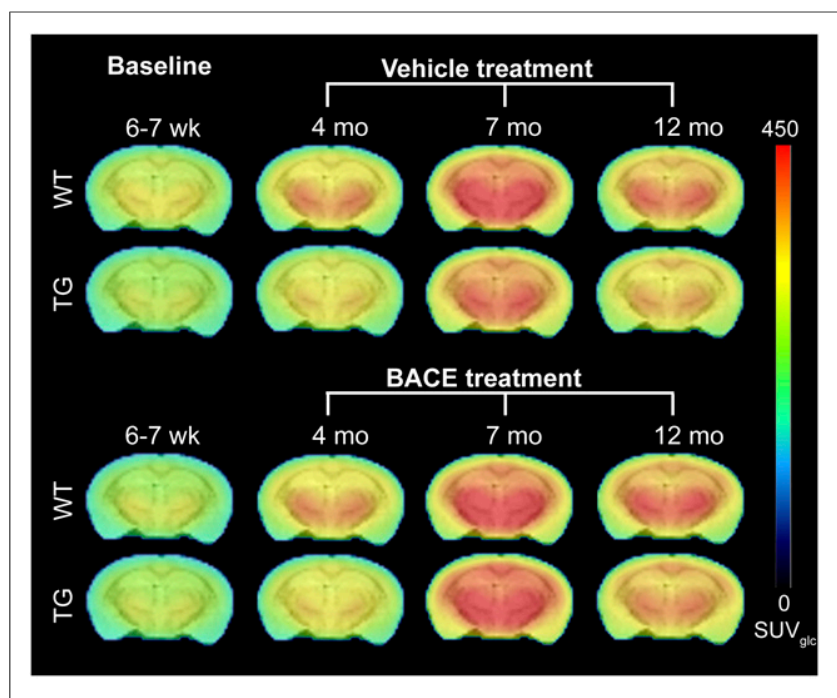

FIGURE 3. Average PET images of ${ }^{18} \mathrm{~F}-\mathrm{FDG}$ uptake in all animal groups, overlaid on MRI T2 template for anatomic reference.

genotype $\times$ treatment interaction were found, indicating that BACE treatment did not influence ${ }^{18}$ F-FDG uptake.

As shown in Figure 4C, TG mice demonstrated lower NeuN staining, and genotype was found to have a significant effect in the cortex $(P<0.0001)$ and thalamus $(P=0.0160)$. A weak negative relationship was found between ${ }^{18} \mathrm{~F}$-FDG uptake and NeuN staining in TG mice $(r=-0.2219, P=0.0136)$.
Representative images of NeuN staining in TG mice are shown in Figure 4D.

\section{${ }^{18}$ F-PBR111 Imaging}

Figure 5 shows the average ${ }^{18} \mathrm{~F}-\mathrm{PBR} 111$ uptake per animal group for each time point. Graphical analysis of ${ }^{18} \mathrm{~F}-\mathrm{PBR} 111 \mathrm{up}-$ take is shown in Figures 6A and 6B. A significant genotype $\times$ age interaction was detected in all brain regions, with TG mice demonstrating increased ${ }^{18} \mathrm{~F}-\mathrm{PBR} 111$ uptake with age $(P<0.0001)$. No effect of treatment was detected.

The TG groups did not differ in total number of microglia (Supplemental Fig. 3). When resting microglia were excluded from Iba-1 analysis, TG-BACE demonstrated a significantly lower number of activated microglia in the cortex $(P=0.0353$, Cohen $\mathrm{d}=0.6670)$, thalamus $(P=0.0096$, Cohen $\mathrm{d}=0.8359)$, and hippocampus $(P=0.0006$, Cohen $\mathrm{d}=1.1419)$ than TG-VEH mice (Fig. 6C). ${ }^{18}$ F-PBR111 demonstrated a low positive correlation with ex vivo Iba-1 measurements of activated microglia $(r=0.3435, P<0.0001)$. Representative images of Iba-1 staining in TG mice are shown in Figure 6D. The effect sizes for differences in ${ }^{18} \mathrm{~F}-\mathrm{PBR} 111$ uptake between TG-VEH and TG-BACE mice at 12 mo were considerably lower than those shown by histology, with Cohen $d=0.1254$ and 0.1941 for the thalamus and hippocampus, respectively. GFAP levels were significantly higher in TG-VEH mice than TG-BACE mice in the cortex $(P=0.0318)$, thalamus $(P=0.0245)$, and hippocampus $(P=0.0318)$ (Supplemental Fig. 4A). ${ }^{18}$ F-PBR111 uptake demonstrated a weak negative correlation with GFAP staining $(r=-0.1275, P=0.1549)$ (Supplemental Fig. 4B).

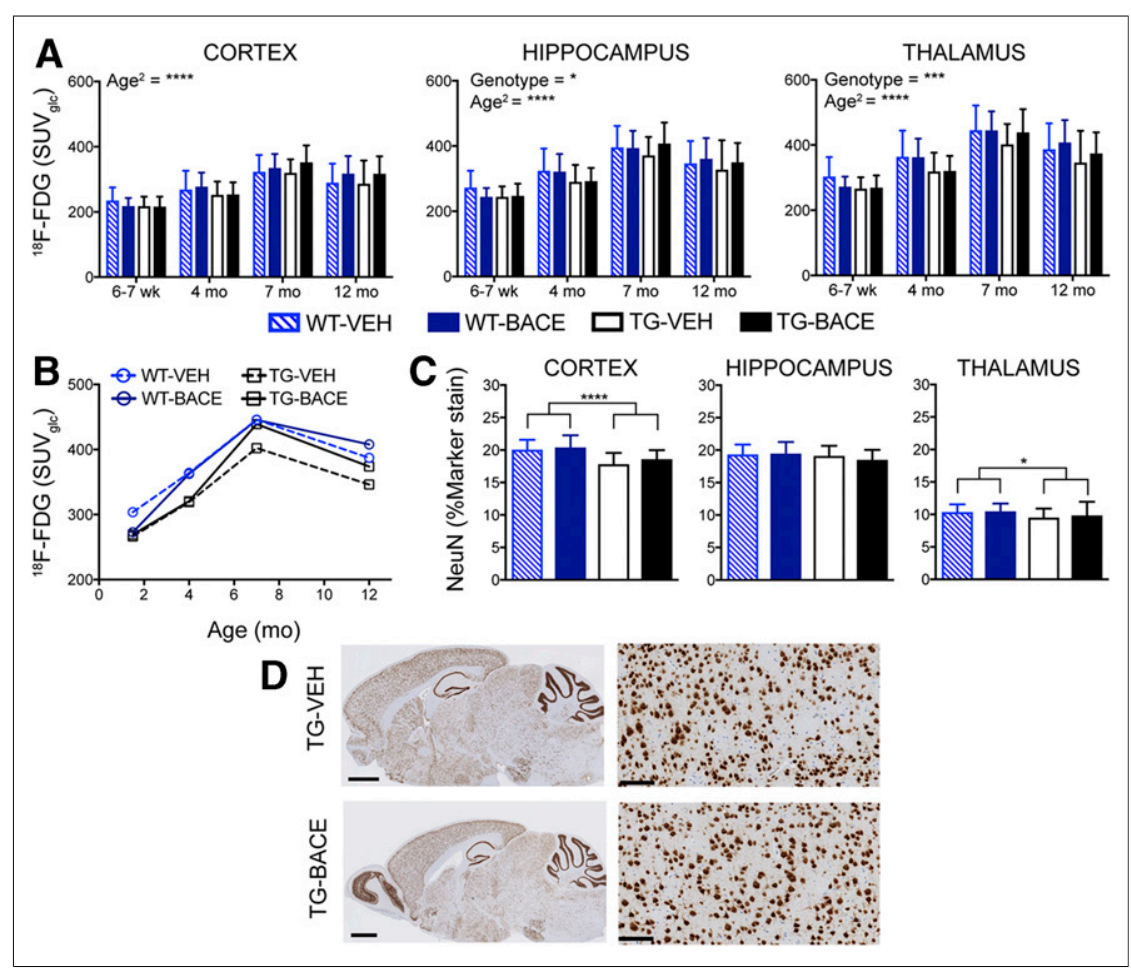

FIGURE 4. Progression of ${ }^{18} \mathrm{~F}-\mathrm{FDG}$ uptake and its relationship to reduced NeuN staining. (A) ${ }^{18} \mathrm{~F}-\mathrm{FDG}$ uptake (mean $+\mathrm{SD}$ ) in cortex, hippocampus, and thalamus. (B) Longitudinal trajectory of mean ${ }^{18}$ F-FDG uptake in thalamus. (C) NeuN staining for neuronal nuclei (mean + SD) in cortex, hippocampus, and thalamus. (D) Representative images of NeuN staining in TG mice. Scale bar $=1.25 \mathrm{~mm}$ (left) and $100 \mu \mathrm{m}$ (right: cortex). ${ }^{\star} P<0.05 ;{ }^{\star \star} P<0.01$; ${ }^{\star \star} P<0.001$; ${ }^{\star \star \star \star} P<0.0001$.

\section{DISCUSSION}

In this study, we investigated the effects of chronic administration of a BACE inhibitor on Alzheimer disease-related pathology by multitracer small-animal PET imaging in TG mice.

${ }^{18} \mathrm{~F}-\mathrm{AV} 45$ has been implemented in animal models to monitor changes in amyloid pathology with age $(30,31)$. It has been shown that other amyloid tracers, such as ${ }^{11} \mathrm{C}$-PiB and ${ }^{18} \mathrm{~F}$-florbetaben, are capable of detecting a treatment effect with amyloid-modulating therapies in transgenic mouse models $(18,19) .{ }^{18} \mathrm{~F}-\mathrm{AV} 45$ uptake was higher in TG mice and increased with age. A treatment effect was observed; however, it was not specific to TG mice. At $8 \mathrm{mo},{ }^{18} \mathrm{~F}-\mathrm{AV} 45$ uptake in WT-BACE mice was lower than that in WT-VEH mice. These findings are anomalous, as WT mice do not develop plaque pathology. ${ }^{18} \mathrm{~F}-\mathrm{AV} 45$ demonstrates high nonspecific binding; for this reason, normalization of regional tracer uptake to a reference region is recommended. Although the cerebellum in TG mice displayed minimal plaque pathology, ${ }^{18} \mathrm{~F}-\mathrm{AV} 45$ uptake was higher in TG mice than WT mice, and cerebellum thus could not be used as a reference region. The 


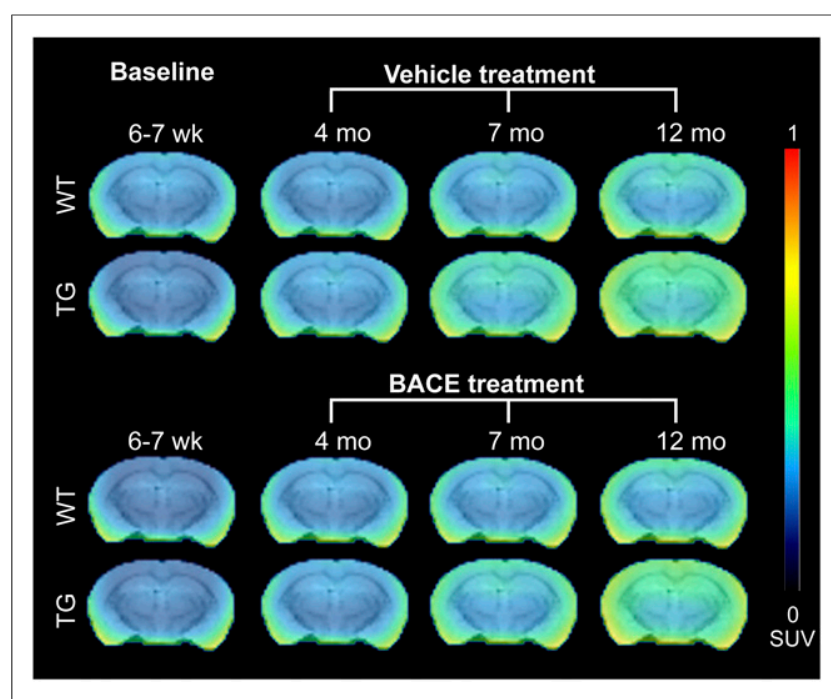

FIGURE 5. Average PET images of ${ }^{18} \mathrm{~F}-\mathrm{PBR} 111$ uptake in all animal groups, overlaid on MRI T2 template for anatomic reference.

significant genotypic differences in ${ }^{18} \mathrm{~F}$-AV45 uptake in TG mice in this study contrast with our previous investigation, which did not find significant differences in ${ }^{18} \mathrm{~F}$-AV45 uptake in 12 -mo-old TG mice. This difference is likely attributable to the higher number of animals in the current study (32).

Replication of the hypometabolic phenotype with smallanimal imaging appears highly dependent on the animal model used. We previously demonstrated hypometabolism in 12-mo-old TG mice with in vivo ${ }^{18} \mathrm{~F}-\mathrm{FDG}$ imaging, confirmed by ex vivo ${ }^{14} \mathrm{C}$-2-deoxyglucose autoradiography (32). Assuming that hypometabolism is a downstream consequence of amyloid- $\beta$ pathology, we hypothesized that reductions in ${ }^{18} \mathrm{~F}$-FDG in TG mice would worsen with increasing age and that lowering amyloid- $\beta$ pathology would prevent or slow changes in brain metabolism. Although ${ }^{18} \mathrm{~F}-\mathrm{FDG}$ uptake was significantly reduced in the thalamus and hippocampus of TG mice, ${ }^{18}$ F-FDG uptake actually increased with age. Takkinen et al. also performed a longitudinal investigation of ${ }^{18} \mathrm{~F}-\mathrm{FDG}$ uptake in TG mice at 6,12 , and 15 mo of age. They similarly found reduced ${ }^{18} \mathrm{~F}$-FDG uptake in TG mice in comparison to WT and an age-dependent increase in ${ }^{18} \mathrm{~F}$-FDG uptake in both genotypes (33). In a previous longitudinal investigation of the double transgenic APPswe $\times$ PSI.MI46V mouse model, we also reported a lack of association between worsening amyloid- $\beta$ pathology and reductions in ${ }^{18} \mathrm{~F}$-FDG uptake (34). It is possible that the observed hypometabolism in these models is a result of neurodevelopmental changes induced by transgene expression and that, in this way, altered brain metabolism would be independent of changes in amyloid- $\beta$ levels. Regarding the lack of treatment effect on ${ }^{18}$ F-FDG response, it should be noted that improvements in ${ }^{18}$ F-FDG uptake through amyloid- $\beta$ reduction have not been demonstrated in human Alzheimer disease. Several approved symptomatic therapies, such as cholinesterase inhibitors, have been shown to influence ${ }^{18} \mathrm{~F}-\mathrm{FDG}$ uptake in patients $(35,36)$, and investigation of ${ }^{18} \mathrm{~F}-\mathrm{FDG}$ as an outcome measure in response to such therapies in amyloid precursor protein models would provide further insight into the utility of this tracer as a preclinical biomarker. Moreover, ${ }^{18}$ F-FDG imaging detected age-dependent decreases in the tauopathy mouse model tauVLW (37) and thus may be more applicable in models with tau pathology. Because activated glial cells also take up ${ }^{18} \mathrm{~F}-\mathrm{FDG}$, we additionally investigated the relationship between ${ }^{18} \mathrm{~F}$-FDG and inflammatory markers and found a weak negative relationship between in vivo ${ }^{18} \mathrm{~F}-\mathrm{FDG}$ uptake and both Iba-1 and GFAP staining (Supplemental Figs. 5A and 5B). Because brain metabolism is the summation of numerous processes, an ex vivo correlate for ${ }^{18} \mathrm{~F}-\mathrm{FDG}$ imaging is difficult. Here, we considered neuronal loss as a potential mechanism underlying hypometabolism. Regardless of treatment, TG mice demonstrated lower NeuN staining than WT mice. Given the trajectory of ${ }^{18} \mathrm{~F}-\mathrm{FDG}$ uptake in TG mice and the lack of correlation between NeuN staining and ${ }^{18} \mathrm{~F}$-FDG uptake, it is highly unlikely that neuronal loss mediates the observed hypometabolism. Quantification of NeuN staining was performed by a nonstereologic method, which has several limitations. Use of unbiased stereologic methods would have provided higher levels of sensitivity and accuracy in determining neuronal numbers (38).

The translocator protein radioligands ${ }^{18} \mathrm{~F}-\mathrm{GE} 180,{ }^{11} \mathrm{C}-\mathrm{PBR} 28$, and ${ }^{18} \mathrm{~F}-\mathrm{DPA}-714$
FIGURE 6. Progression of ${ }^{18} \mathrm{~F}-\mathrm{PBR} 111$ uptake and its relationship to lba-1 staining (A) ${ }^{18} \mathrm{~F}-\mathrm{PBR} 111$ uptake (mean $+\mathrm{SD}$ ) in cortex, hippocampus, and thalamus. (B) Longitudinal trajectory of mean ${ }^{18} \mathrm{~F}-\mathrm{PBR} 111$ uptake in thalamus. (C) Iba-1 staining for microglia (mean + SD) in cortex, hippocampus, and thalamus. (D) Representative images of Iba-1 staining in TG mice. Scale bar $=1.25 \mathrm{~mm}$ (left) and $100 \mu \mathrm{m}$ (right: cortex). ${ }^{\star} P<0.05 ;{ }^{\star \star} P<0.01$; ${ }^{\star \star \star} P<0.001 ;{ }^{\star \star \star \star} P<0.0001$. 
have demonstrated age-related increases in PS2APP, APPPS1d9, 5xFAD, and TG mouse models $(33,39)$. Additionally, chronic BACE inhibitor treatment (NB-360) has previously been shown to reduce glial cells in APP51/16 mice (12), and thus we herein evaluated the ability of ${ }^{18} \mathrm{~F}-\mathrm{PBR} 111$ to detect changes in neuroinflammation in response to disease and treatment. In this study, we found a significant genotype $\times$ age interaction on ${ }^{18} \mathrm{~F}-\mathrm{PBR} 111$ uptake in the cortex, thalamus, and hippocampus, indicating the ability of this tracer to monitor worsening inflammation with disease progression. This finding agrees with a previous demonstration of age- and genotyperelated increases in ${ }^{18} \mathrm{~F}$-DPA-714 binding in TG mice (33). However, the correlation between ${ }^{18} \mathrm{~F}-\mathrm{PBR} 111$ uptake and the microglial marker Iba-1 was only moderate, and in vivo imaging did not detect the reduction of activated microglia in BACEtreated TG mice demonstrated by histology. The poor sensitivity of ${ }^{18} \mathrm{~F}-\mathrm{PBR} 111$ could be due to the release of ${ }^{18} \mathrm{~F}$-fluoride during its metabolism (40), as uptake of ${ }^{18} \mathrm{~F}$-fluoride by skull bone greatly complicates tracer quantification in brain tissue. Because activated astrocytes can also bind translocator protein radioligands, the poor correlation between Iba-1 and ${ }^{18}$ F-PBR111 could be due to astrocytic binding of ${ }^{18} \mathrm{~F}-\mathrm{PBR} 111$. However, we found no correlation between GFAP staining and ${ }^{18} \mathrm{~F}-\mathrm{PBR} 111$, indicating that activated microglia likely underlie the increased retention of ${ }^{18} \mathrm{~F}-\mathrm{PBR} 111$ in TG mice. The translocator protein radioligands ${ }^{18} \mathrm{~F}-\mathrm{GE} 180$ and ${ }^{11} \mathrm{C}$-PBR28 have demonstrated strong correlations with histologic measures of neuroinflammation, additionally demonstrated increased retention at young ages (5-6 mo) in transgenic mice $(38,41)$, and may thus be more suitable for small-animal imaging.

Considering the multitracer and longitudinal design of the current study, we used static PET scans to minimize scan duration and anesthesia exposure. As a result, tracer uptake was quantified by semiquantitative methods, which have several drawbacks (42). However, absolute quantification or reference tissue modeling was not feasible given that the use of plasma-derived input functions is not possible for longitudinal studies and suitable region references were not available.

\section{CONCLUSION}

${ }^{18} \mathrm{~F}-\mathrm{AV} 45,{ }^{18} \mathrm{~F}-\mathrm{FDG}$, and ${ }^{18} \mathrm{~F}-\mathrm{PBR} 111$ detected pathologic differences between WT and TG mice. Only ${ }^{18} \mathrm{~F}$-AV45 could detect a treatment-induced reduction in pathology. However, changes in WT binding of ${ }^{18} \mathrm{~F}-\mathrm{AV} 45$ undermine the specificity of this effect. For ${ }^{18}$ F-FDG, it is highly unlikely that neuronal loss mediates the observed hypometabolism, and ${ }^{18}$ F-PBR 111 was not sensitive enough to detect a treatment effect, likely because of radioactivity uptake in the skull bone.

\section{DISCLOSURE}

This work was funded by Antwerp University and its University Hospital, Antwerp, Belgium, through a postdoctoral research position for Steven Deleye, an assistant professor position for Leonie Wyffels and Jeroen Verhaeghe, an associate professor position for Steven Staelens, and a full professor position for Sigrid Stroobants. Ann-Marie Waldron is supported through an IWT scholarship (Agency for
Innovation by Science and Technology). Astrid Bottelbergs, Xavier Langlois, and Mark Schmidt are with Janssen Pharmaceutica. No other potential conflict of interest relevant to this article was reported.

\section{ACKNOWLEDGMENTS}

We thank Philippe Joye (Molecular Imaging Center Antwerp) and Caroline Berghmans for support with in vivo experiments, Dr. José Ignacio Andrés (Janssen Research and Development) for preparation of the AV-105 cold reference, Dr. Hilde Vermeirsch (Histogenex) for ex vivo validation processing, and Erik Franssen for statistical assistance.

\section{REFERENCES}

1. Selkoe DJ. The molecular pathology of Alzheimer's disease. Neuron. 1991;6:487-498.

2. Svedberg MM, Rahman O, Hall H. Preclinical studies of potential amyloid binding PET/ SPECT ligands in Alzheimer's disease. Nucl Med Biol. 2012;39:484-501.

3. Casellas P, Galiegue S, Basile AS. Peripheral benzodiazepine receptors and mitochondrial function. Neurochem Int. 2002;40:475-486.

4. Mosconi L. Brain glucose metabolism in the early and specific diagnosis of Alzheimer's disease: FDG-PET studies in MCI and AD. Eur J Nucl Med Mol Imaging. 2005;32:486-510.

5. Kobayashi N, Ueda K, Itoh N, et al., inventors. Shionogi and Co., Ltd., assignee. Pharmaceutical composition for treatment of Alzheimer's disease. Patent WO2008133273 A1. April 23, 2008.

6. Laird FM. BACE1, a major determinant of selective vulnerability of the brain to amyloid- $\beta$ amyloidogenesis, is essential for cognitive, emotional, and synaptic functions. J Neurosci. 2005;25:11693-11709.

7. Devi L, Ohno M. Effects of BACE1 haploinsufficiency on APP processing and Ab concentrations in male and female 5XFAD Alzheimer mice at different disease stages. Neuroscience. 2015;307:128-137.

8. Kimura R, Devi L, Ohno M. Partial reduction of BACE1 improves synaptic plasticity, recent and remote memories in Alzheimer's disease transgenic mice. J Neurochem. 2010;113:248-261.

9. Fukumoto H, Takahashi H, Tarui N, et al. A noncompetitive BACE1 inhibitor TAK-070 ameliorates $A \beta$ pathology and behavioral deficits in a mouse model of Alzheimer's disease. J Neurosci. 2010;30:11157-11166.

10. Devi L, Tang J, Ohno M. Beneficial effects of the $\beta$-secretase inhibitor GRL-8234 in 5XFAD Alzheimer's transgenic mice lessen during disease progression. Curr Alzheimer Res. 2015;12:13-21.

11. Thakker DR, Sankaranarayanan S, Weatherspoon MR, et al. Centrally delivered BACE1 inhibitor activates microglia, and reverses amyloid pathology and cognitive deficit in aged Tg2576 mice. J Neurosci. 2015;35:6931-6936.

12. Neumann U, Rueeger H, Machauer R, et al. A novel BACE inhibitor NB-360 shows a superior pharmacological profile and robust reduction of amyloid- $\beta$ and neuroinflammation in APP transgenic mice. Mol Neurodegener. 2015;10:44.

13. Eketjäll S, Janson J, Jeppsson F, et al. AZ-4217: a high potency BACE inhibitor displaying acute central efficacy in different in vivo models and reduced amyloid deposition in Tg2576 mice. J Neurosci. 2013;33:10075-10084.

14. Vassar R. BACE1 inhibitor drugs in clinical trials for Alzheimer's disease. Alzheimers Res Ther. 2014;6:89.

15. Radde R, Bolmont T, Kaeser SA, et al. A 342 -driven cerebral amyloidosis in transgenic mice reveals early and robust pathology. EMBO Rep. 2006;7:940-946.

16. Serneels L, Van Biervliet J, Craessaerts K, et al. y-secretase heterogeneity in the Aph1 subunit: relevance for Alzheimer's disease. Science. 2009;324:639-642.

17. Bittner T, Burgold S, Dorostkar MM, et al. Amyloid plaque formation precedes dendritic spine loss. Acta Neuropathol (Berl). 2012;124:797-807.

18. Maeda J, Ji B, Irie T, et al. Longitudinal, quantitative assessment of amyloid, neuroinflammation, and anti-amyloid treatment in a living mouse model of Alzheimer's disease enabled by positron emission tomography. J Neurosci. 2007;27:10957-10968.

19. Brendel M, Jaworska A, Herms J, et al. Amyloid-PET predicts inhibition of de novo plaque formation upon chronic $\gamma$-secretase modulator treatment. $\mathrm{Mol}$ Psychiatry. 2015;20:1179-1187.

20. Rombouts FJR, Tresadern G, Delgado O, et al. 1,4-oxazine $\beta$-secretase 1 (BACE1) inhibitors: from hit generation to orally bioavailable brain penetrant leads. J Med Chem. 2015;58:8216-8235. 
21. Yao C-H, Lin W-L, Weng C-C, et al. GMP-compliant automated synthesis of $\left[{ }^{18} \mathrm{~F}\right] \mathrm{AV}-45$ (Florbetapir F 18) for imaging beta-amyloid plaques in human brain. Appl Radiat Isot. 2010;68:2293-2297.

22. Bourdier T, Pham TQ, Henderson $D$, et al. Automated radiosynthesis of $\left[{ }^{18} \mathrm{~F}\right]$ PBR111 and $\left[{ }^{18}\right.$ F]PBR102 using the Tracerlab FXFN and Tracerlab MXFDG module for imaging the peripheral benzodiazepine receptor with PET. Appl Radiat Isot. 2012;70:176-183.

23. Kemp BJ, Hruska CB, McFarland AR, Lenox MW, Lowe VJ. NEMA NU 2-2007 performance measurements of the Siemens Inveon preclinical small animal PET system. Phys Med Bio. 2009;54:2359-2376.

24. Hudson HM, Larkin RS. Accelerated image reconstruction using ordered subsets of projection data. IEEE Trans Med Imaging. 1993;13:601-609.

25. Defrise M, Kinahan PE, Townsend DW, Michel C, Sibomana M, Newport DF. Exact and approximate rebinning algorithms for 3-D PET data. IEEE Trans Med Imaging. 1997;16:145-158.

26. Watson CC. New, faster, image-based scatter correction for 3D PET. IEEE Trans Nucl Sci. 2000;47:1587-1594.

27. Mirrione MM, Schiffer WK, Fowler JS, Alexoff DL, Dewey SL, Tsirka SE. A novel approach for imaging brain-behavior relationships in mice reveals unexpected metabolic patterns during seizures in the absence of tissue plasminogen activator. Neuroimage. 2007;38:34-42.

28. Franklin KB, Paxinos G. The Mouse Brain in Stereotaxic Coordinates. Cambridge, MA: Academic Press; 1997.

29. Shimshek DR, Jacobson LH, Kolly C, et al. Pharmacological BACE1 and BACE2 inhibition induces hair depigmentation by inhibiting PMEL17 processing in mice. Sci Rep. 2016;6:21917.

30. Sérrière $\mathrm{S}$, Tauber $\mathrm{C}$, Vercouillie $\mathrm{J}$, et al. Amyloid load and translocator protein $18 \mathrm{kDa}$ in APPswePS1-dE9 mice: a longitudinal study. Neurobiol Aging. 2015;36:1639-1652.

31. Poisnel G, Dhilly M, Moustié O, et al. PET imaging with $\left[{ }^{18} \mathrm{~F}\right] \mathrm{AV}-45$ in an APP/PS1-21 murine model of amyloid plaque deposition. Neurobiol Aging. 2012;33:2561-2571.
32. Waldron A-M, Wintmolders C, Bottelbergs A, et al. In vivo molecular neuroimaging of glucose utilization and its association with fibrillar amyloid- $\beta$ load in aged APPPS1-21 mice. Alzheimers Res Ther. 2015;7:76.

33. Takkinen JS, Lopez-Picon FR, Al Majidi R, et al. Brain energy metabolism and neuroinflammation in ageing APP/PS1-21 mice using longitudinal ${ }^{18} \mathrm{~F}-\mathrm{FDG}$ and ${ }^{18}$ F-DPA-714 PET imaging. J Cereb Blood Flow Metab. 2017;37:2870-2882.

34. Waldron AM, Wyffels L, Verhaeghe J, et al. Longitudinal characterization of $\left[{ }^{18} \mathrm{~F}\right]-\mathrm{FDG}$ and $\left[{ }^{18} \mathrm{~F}\right]-\mathrm{AV} 45$ uptake in the double transgenic TASTPM mouse model. J Alzheimers Dis. 2017;55:1537-1548.

35. Stefanova E, Wall A, Almkvist O, et al. Longitudinal PET evaluation of cerebral glucose metabolism in rivastigmine treated patients with mild Alzheimer's disease. J Neural Transm. 2006;113:205-218.

36. Mega MS, Dinov ID, Porter V, et al. Metabolic patterns associated with the clinical response to galantamine therapy: a fludeoxyglucose f 18 positron emission tomographic study. Arch Neurol. 2005;62:721-728.

37. de Cristóbal J, García-García L, Delgado M, Pérez M, Pozo MA, Medina M. Longitudinal assessment of a transgenic animal model of tauopathy by FDG-PET imaging. J Alzheimers Dis. 2014;40(suppl 1):S79-S89.

38. Howard V, Reed MG. Unbiased Stereology: Three-Dimensional Measurement in Microscopy. New York, NY: Garland Science/BIOS Scientific Publishers; 2005.

39. Brendel M, Probst F, Jaworska A, et al. Glial activation and glucose metabolism in a transgenic amyloid mouse model: a triple-tracer PET study. J Nucl Med. 2016;57:954-960.

40. Fookes CJR, Pham TQ, Mattner F, et al. Synthesis and biological evaluation of substituted $\left[{ }^{18} \mathrm{~F}\right]$ imidazo $[1,2-a]$ pyridines and $\left[{ }^{18} \mathrm{~F}\right]$ pyrazolo $[1,5-a]$ pyrimidines for the study of the peripheral benzodiazepine receptor using positron emission tomography. J Med Chem. 2008;51:3700-3712.

41. Mirzaei N, Tang SP, Ashworth S, et al. In vivo imaging of microglial activation by positron emission tomography with $\left[{ }^{11} \mathrm{C}\right] \mathrm{PBR} 28$ in the 5XFAD model of Alzheimer's disease. Glia. 2016;64:993-1006.

42. Keyes JW Jr. SUV: standard uptake or silly useless value? J Nucl Med. 1995;36:1836-1839. 